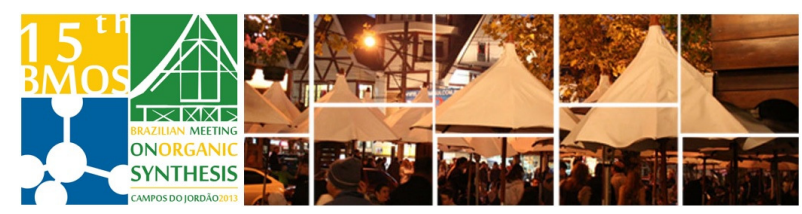

\title{
Application of a biarylarsine ligand in Heck and Stille reactions
}

\author{
Quinteros, Gisela J.; Uberman, Paula M. and Martín, Sandra E.* \\ INFIQC, Departamento de Química Orgánica, Facultad de Ciencias Químicas, Universidad Nacional de \\ Córdoba, 5000-Córdoba, Argentina. \\ *e-mail martins@fcq.unc.edu.ar
}

Keywords: biarylarsine ligand, Heck reaction, Stille reaction

\section{INTRODUCTION}

The Pd coupling reactions are widely used in organic synthesis. Among this group, the Heck reaction occupies a main role in the arylation of alkenes, with wide application in the synthesis of natural products. ${ }^{1}$ Moreover, the Stille reaction is a powerful method in organic synthesis due to its broad functional group tolerance and the stability of the reagents. ${ }^{2}$

In the last years, developments in coupling reactions are based on designing selective and stable ligands. Although phosphines ligands play a preponderant role in this field, arsines have shown to be excellent

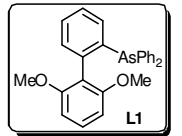

Figure 1 supporting ligands, ${ }^{3}$ but relatively few have been prepared and applied in catalysis. Recently we have developed a family of biarylarsine ligands that show excellent activity in Stille reaction. ${ }^{4}$ Herein we report the evaluation of bulky arsine L1 (Figure 1) in Stille and Heck reactions.

\section{RESULTS AND DISCUSSION}

Based on initial screening, a combination of L1 with $\mathrm{Pd}(\mathrm{OAc})_{2}$ generate a highly active catalyst system for Heck and Stille reactions.

In order to establish the scope of the catalytic system for the Heck reaction (Scheme 1), several aryl iodides and aryl bromides were evaluated with styrene and $n$-butyl acrylate as the coupling partner. For substrates with electron withdrawing groups the conversion was complete in very short time with excellent yields. In the case of electrophiles with electron donating substituents, good yields were obtained but the reaction did not finish until $24 \mathrm{~h}$. The best results were obtained employing $1 \mathrm{~mol} \%$ $\mathrm{Pd}(\mathrm{OAc})_{2}, 1: 2$ ratio $\mathrm{Pd}: \mathrm{L} 1,1$ eq. of the aryl halide, 1.5 eq. of styrene, 2 eq. of $\mathrm{K}_{2} \mathrm{CO}_{3}$ as a base in DMF as solvent at $140^{\circ} \mathrm{C}$ (Scheme 1 ).

Arsine L1 was also evaluated in the Stille reaction (Scheme 2). Among all the reaction conditions screened, the best results were found by using 1 mol\% $\mathrm{Pd}(\mathrm{OAc})_{2}, 1: 2$ ratio $\mathrm{Pd}: \mathbf{L 1}, 1$ eq. of the aryl halide, $1.1 \mathrm{eq}$. of stannane, $2.2 \mathrm{eq}$. of CsF, using dioxane as solvent at $80^{\circ} \mathrm{C}$. The Pd-L1 complex was screened with several arylbromides and different stannane reagents.

$15^{\text {th }}$ Brazilian Meeting on Organic Synthesis $-15^{\text {th }}$

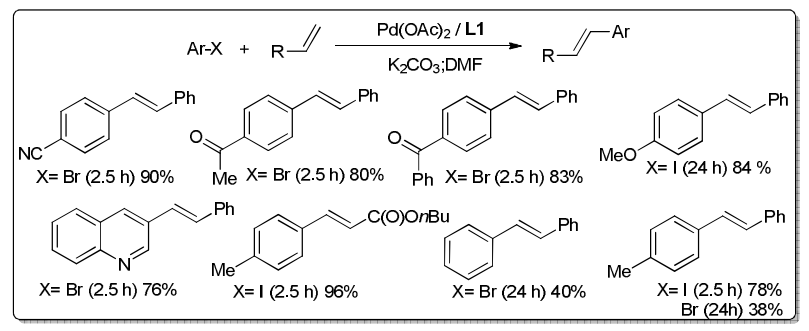

Scheme 1. L1 in Heck reaction.

By this way it was possible to obtain good and excellent yields of biaryl and arilvinyl products (Scheme 2).

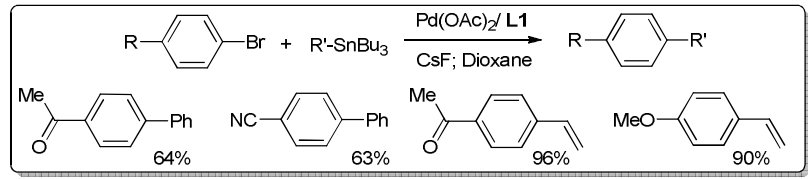

Scheme 2. L1 in Stille reaction.

A novel two step one-pot methodology combining a Stille-Heck sequence catalyzed with Pd-L1 was developed. By this approach the substituted stilbene 2 was obtained with excellent yields (Scheme 3 ).

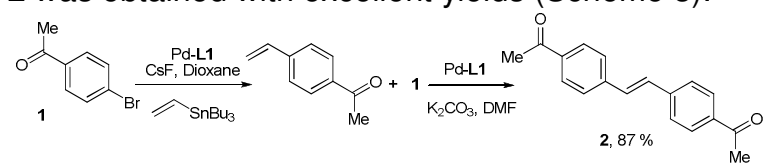

Scheme 3. One-pot Stille-Heck sequence for stilbene synthesis.

\section{CONCLUSION}

The catalytic activity of the complex Pd-arsine (L1) was evaluated in Heck and Stille reactions, obtaining very good yields of the coupling products. These promising results encouraged us to develop a novel approach for the synthesis of stilbene compounds.

\section{ACKNOWLEDGEMENTS}

We are grateful to CONICET, FONCYT and SECYTUNC for financial support.

\section{REFERENCES}

${ }_{2}^{1}$ Nadri, S.; Joshaghani, M.; Rafiee, E. App. Cat. A, 2009, 362, 163.

${ }^{2}$ Naber, J.; Buchwald, S. Adv. Synth. Catal. 2008, 350, 957.

${ }^{3}$ Baber, R.; Collard, S.; Hooper, M.; Orpen, A.; Pringle, P.; Wilkinson, M.; Wingad, R. Dalton Trans. 2005, 1491.

${ }^{4}$ Uberman, P.; Lanteri, M.; Parajón Puenzo, S.; Martín, S. Dalton Trans. $2011,40,9229$. 